\title{
LAS RELACIONES INTRAPERSONALES E INTERPERSONALES EN ESTUDIANTES DESVINCULADOS DEL CONFLICTO ARMADO COLOMBIANO E INCLUIDOS EN EL AULA DEL SOL
}

\author{
Oscar Andrés Alzate Mejía ${ }^{1}$
}

Manizales, 2009-10-12 (Rev. 2009-11-25)

\begin{abstract}
RESUMEN
El Ministerio de Educación Nacional y el Instituto Colombiano de Bienestar Familiar (ICBF) establecieron y desarrollaron los planes necesarios para el restablecimiento de los derechos y garantías del menor desvinculado del conflicto armado, con especial énfasis en la educación. En la ciudad de Manizales, la Universidad de Caldas conformó un equipo de trabajo que lidera el proceso de vinculación y formación de estos jóvenes en el sistema educativo.
\end{abstract}

En el presente artículo se mostrará la influencia que tuvo la asignatura de Ecología Humana en las relaciones intrapersonales e interpersonales de los estudiantes desvinculados del conflicto armado colombiano e incluidos en el aula del sol de la ciudad de Manizales.

La metodología empleada fue de corte cualitativo con enfoque descriptivo comprensivo. El estudio se llevó a cabo en el aula del sol que es el espacio educativo para jóvenes desvinculados de los grupos armados el cual funciona en una institución educativa de Manizales como un aula multigrado.

Los resultados de esta investigación fueron satisfactorios, los datos obtenidos permitieron reconocer claramente la influencia de la ecología humana en la transformación de las relaciones de los estudiantes del aula del sol. Dicho de otra manera, el aula del sol es una hermosa mañana, la ecología humana son los rayos de luz que calientan e iluminan momentos de adaptación, reflexión y orientación; se visiona que como en las plantas, se transforme esa luz solar en frutos para el bien de sí mismo y de su entorno.

\section{PALABRAS CLAVE}

Ecología humana, ecosistema humano, ambiente interpersonal, nicho afectivo, conflicto armado.

\section{INTRAPERSONAL AND INTERPERSONAL RELATIONS IN DISARMED STUDENTS OF THE COLOMBIAN ARMED CONFLICT AND INCLUDED IN THE "SUN CLASSROOM"}

\begin{abstract}
The National Education Ministry and the Colombian Institute for Family Welfare (ICBF) established and developed the necessary plans for the restoration of the rights and guarantees of disengaged disarmed children of the armed conflict, with special emphasis on education. In the city of Manizales, the Universidad de Caldas University formedestablished a work team to leading the process of linking and educating on of these young people with in the education system. This article shows the influence that the subject course titledof "hHuman eEcology" had ion intrapersonales and interpersonal relations ion disarmed students unliked offrom the Colombian armed conflict and included in the "Sun cClassroom" of city of located in Manizales. The qualitative methodology was implemented qualitative with a comprehensive descriptive approach. The study was carried out in the "Sun cClassroom", that is an educational space for detacheddisarmed youth, of armed groups which works operates in the a Manizales high school of Manizales as a multigrade classroom. The results of this research were satisfactory, since they obtained data permit clearly evidencedrecognize the influence of Human Ecology in the transformation of the relations of among the "Sun cClassroom"s" students. Another wayln other words, the "Sun cClassroom" is a beautiful morning, ecology hHuman Ecology are rays of light that heatedwarm and light moments of adaptation, reflection and orientation moments; to see thatand, as it happens with in plants, to transform that sunlight is transformed in fruits for the good of itself and its environment.
\end{abstract}


KEY WORDS: Human Ecology, human ecosystem, interpersonal ambientenvironment, affective niche, armed conflict.

\section{INTRODUCCIÓN}

Es conocido que Colombia a lo largo de su historia ha tenido una larga y variada gama de violencia. Hoy, después de casi 50 años de insurgencia y 30 años de paramilitarismo se continúa desarrollando un conflicto armado donde se han visto involucrados miles de menores de edad en forma variada: voluntarios, forzados, como víctimas de la violencia o que huyen de sus hogares por problemas o necesidades dentro de sus familias.

En febrero de 2008, la Fundación Maya Nasa reveló que los menores de edad forman una quinta parte de las guerrillas colombianas ${ }^{2}$. Según el estudio, los chicos son usados para hacer explosivos, instalar minas antipersonales, además de hacer labores de ranchería (oficios de cocina y aseo). Uno de los principales trabajos que desempeñan es hacer inteligencia, vigilar y combatir. Estos niños son el primer anillo de seguridad de los grupos armados.

Por lo anterior, resulta necesario re-vincular a la vida social a estos seres humanos, especialmente a los menores de edad que vienen de vivir abusos, maltratos físicos, psicológicos, sexuales, vacíos emocionales y explotación laboral, además de ser necesaria una alianza educativa porque son jóvenes en extraedad, con altas deficiencias en su formación humana y académica. Provienen de condiciones de marginalidad, pobreza extrema y entornos violentos reflejados en sus palabras y acciones.

En el año 2004 el Ministerio de Educación Nacional estableció directrices y criterios para la prestación del servicio educativo a niños, niñas y jóvenes desvinculados del conflicto armado ${ }^{3}$. El Instituto Colombiano de Bienestar Familiar (ICBF) desarrolló los planes necesarios para el restablecimiento de sus derechos. En la ciudad de Manizales, con la Universidad de Caldas desde el CEDAT (Centro de Estudios y Desarrollo Alternativo sobre Territorios de conflicto) y el departamento de Estudios Educativos, se conformó un equipo de trabajo que lidera el proceso de vinculación y formación de estos jóvenes en el sistema educativo con el proyecto aula del sol. El aula del sol es un nuevo amanecer que se presenta dentro de una institución educativa de Manizales para adolescentes desvinculados del conflicto armado colombiano en un aula multigrado de básica primaria.

Las dificultades en la convivencia, los vacíos emocionales, la baja tolerancia a la frustración, la percepción negativa de sí mismo, la desconfianza generalizada, las dificultades en la apropiación y el manejo de la norma, la deprivación afectiva manifiestas en los estudiantes del aula del sol y el interés de sus coordinadores y docentes hicieron surgir la presente investigación. Cómo incidir en las relaciones intrapersonales e interpersonales de los estudiantes del aula del sol de la ciudad de Manizales fue el punto de partida. El propósito, reconocer la influencia de la Ecología Humana en sus relaciones. Así mismo, explicar la importancia del trabajo en equipo y el cambio de actitud de los estudiantes ante las mismas.

La ecología humana fue la herramienta que se aplicó en el proceso, ella se establece como la analogía entre los ecosistemas vivientes y el ecosistema humano. Esta perspectiva se justifica por la similitud que existe entre la crisis ecológica y la crisis interpersonal y valorativa del mundo contemporáneo.

Luis Carlos Restrepo la enfoca tanto como una metodología amplia de reconstrucción cultural e interpersonal, como una perspectiva válida para enfrentar diferentes problemáticas. La ecología humana para él propende por una reconstrucción del espacio cultural y comunicativo, a fin de generar un cambio de actitudes en la esfera de la interpersonalidad.

Por su parte, Tapias y Saavedra la definen como una teoría sobre el Ser Humano (ciencia del amor, la salud, la ética, la comunicación, la convivencia y la felicidad humana) dirigido a su desarrollo, a su transformación y la de su ambiente. La Ecología Humana es un conocimiento aplicable al servicio de la convivencia humana, para recuperar la armonía con el ambiente y devolver el respeto de la ética y de los deberes Humanos. Participa de la crucial campaña por la preservación del planeta y de la 
humanidad. Considera que en tanto el ser humano no sea capaz de cuidar el metro cuadrado que habita, nunca podrá participar con éxito en la preservación de la vida y el ambiente.

La ecología humana es entonces el estudio de las relaciones entre los seres humanos y su entorno, implica el desarrollo y la transformación de ellos mismos y su ambiente. Desde sus objetivos intra e interpersonales, enseña a cuidar nuestros nichos afectivos de la contaminación del ambiente interpersonal. Es una respuesta para ayudar al auténtico restablecimiento, pretende elevar la calidad de vida y la eficiencia para sobrellevar tiempos de cambio, crisis e incertidumbres.

\section{METODOLOGÍA}

La investigación fue hecha en el aula del sol que es el espacio educativo para los jóvenes desvinculados de los grupos armados irregulares provenientes de Hogares Tutores del ICBF. Funciona en una institución educativa de Manizales ${ }^{4}$, su finalidad es mejorar la calidad de vida y generar condiciones propicias y adecuadas para el desarrollo integral de sus estudiantes. Es un aula multigrado de básica primaria constituida a partir de un sistema educativo que promueve el aprendizaje activo, con sistema tutorial personalizado a partir de los diferentes ritmos de trabajo.

El número de estudiantes en el aula tiene un promedio de 20 jóvenes que ingresan en cualquier momento del año de acuerdo a sus necesidades. Después de lograr sus conocimientos de básica primaria o al cumplir su mayoría de edad, ellos avanzan a otras instituciones de formación para adultos y/o de formación técnico-laboral. Para la investigación se trabajó específicamente con los catorce jóvenes que en el momento se encontraban en el aula.

La metodología empleada en esta investigación puede ubicarse en una investigación cualitativa con enfoque descriptivo comprensivo ya que describió y analizó el sentir manifiesto de los estudiantes que conforman el aula del sol acerca de sus relaciones de convivencia, lo cual permitió proponer acciones precisas que mejoraran sus relaciones intrapersonales e interpersonales a partir del concepto de ecología humana.

La metodología de esta investigación se desarrolló en tres momentos específicos:

Inicialmente se hizo un acercamiento a los estudiantes del aula del sol logrando una aproximación y reconocimiento de su contexto, se propició un ambiente favorable de apertura y descubrimiento.

La investigación en su aspecto operativo se desarrolló creando espacios para que cada uno pudiera ser lo que es como ser humano y para que disfrutara con pasión y entusiasmo de un trabajo orientado a recuperar ese poder único y original con el que nace cada ser humano para desarrollarlo al máximo de su potencial. Se orientó en un año y medio y durante dos horas semanales la asignatura de Ecología Humana a través de talleres vivenciales que llevaron a la reflexión y participación de los estudiantes, buscando con la intervención del proceso ecológico un cambio actitudinal y una transformación en las relaciones. Se permitió al estudiante expresar libremente sus ideas y tomar decisiones acordes a la satisfacción de sus necesidades.

Se hizo el análisis y la interpretación de resultados a partir de un diario de campo, dos cuestionarios que respondieron los estudiantes y un cuestionario hecho al equipo coordinador del aula conformado por la docente titular, tres psicólogos, tres trabajadoras sociales, un abogado, una practicante en psicología y otra de desarrollo familiar.

\section{RESULTADOS Y DISCUSIÓN}

Al culminar la investigación se encontró el aula del sol como un espacio que genera relaciones intrapersonales e interpersonales adecuadas. Los compañeros, profesores y el método de enseñanza son las principales razones que hacen al $100 \%$ de los estudiantes disfrutar de su estadía en ella. Los 
estudiantes aprovechan el espacio para reflexionar, opinar, formarse, conocer otras personas y continuar avanzando en el cumplimiento de sus metas.

Se encontró que el $79 \%$ de los estudiantes consideran las relaciones interpersonales en el aula del sol muy buenas y la razón de ser de ello se explica en que trabajan en equipo, se divierten, comparten, se comprenden y se ayudan mutuamente. De otro lado, para el $21 \%$ de los estudiantes las relaciones interpersonales en el aula son regulares, algunos no tienen disposición para escuchar, otros no acatan cumplidamente las normas y existen algunas palabras soeces que indisponen el ambiente grupal (Figura 1 y 2)

Figura 1. Calificación de las relaciones interpersonales de los estudiantes del aula del sol

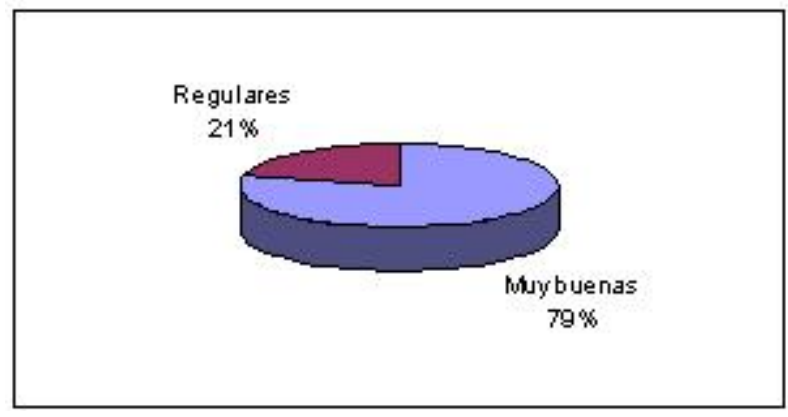

Fuente: Elaboración propia

Figura 2. Razones que explican la calificación de las relaciones interpersonales de los estudiantes del aula del sol

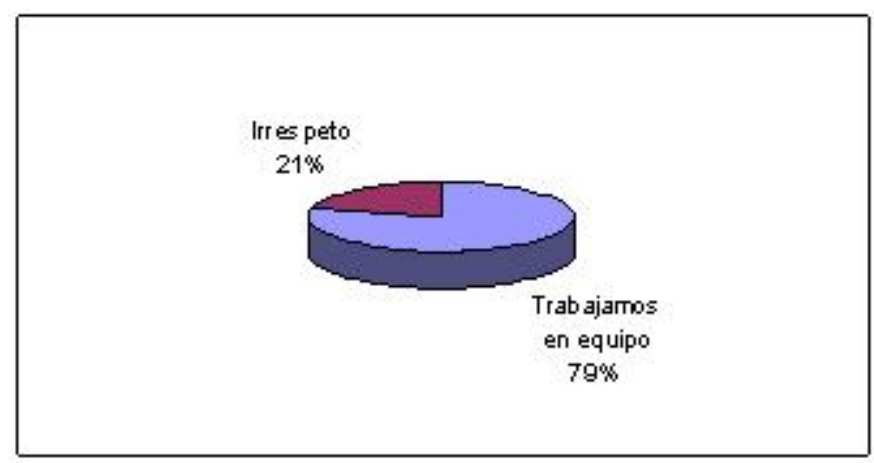

Fuente: Elaboración propia

De la misma forma se encontró que en el 100\% de los estudiantes, la ecología humana mejoró las relaciones con sus compañeros, con sus docentes y con el entorno. La razón por la cual mejoró las relaciones con los compañeros se enmarcó, para el $72 \%$, en el aprendizaje del trabajo en equipo y un $21 \%$ enmarcó el mejoramiento en el respeto, entendido como la importancia que tiene la otra persona (Figura 4). En cuanto a las relaciones con los docentes, la ecología humana en el 50\% ayudó al fortalecimiento de las relaciones estudiante-profesor. El otro $50 \%$ explicó que la ecología humana permitió la ayuda de los docentes en su proceso de ascenso personal y cognitivo. Finalmente, las razones por las que la ecología humana mejoró las relaciones con el entorno, obedecen a que les enseña a cuidar más la naturaleza, el otro 50\% razonó que la ecología humana les ayudó a compartir más con los demás. 
Figura 4. Razones por las que la ecología humana mejora las relaciones con los compañeros

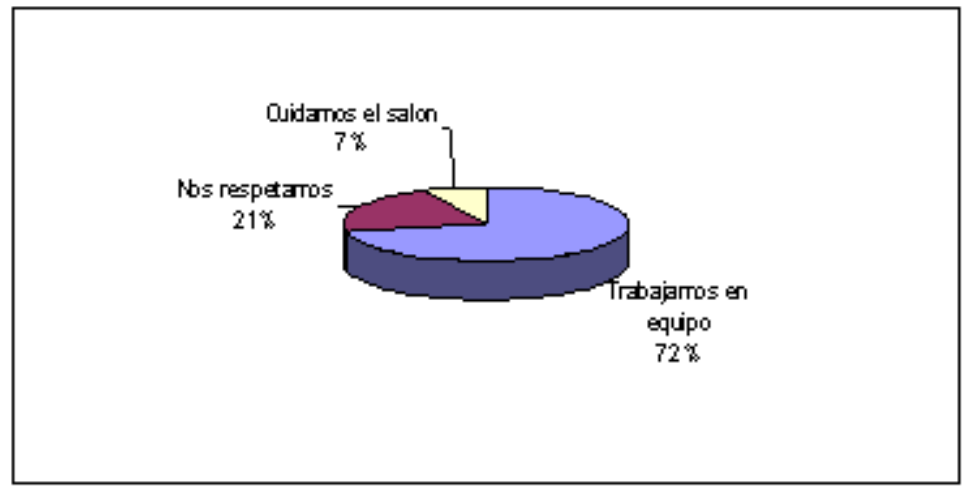

Fuente: Elaboración propia

De igual manera, al analizar los beneficios de los objetivos de la ecología humana en las relaciones intrapersonales e interpersonales es importante observar la apreciación que hicieron los estudiantes, sus mediciones estuvieron por encima de 4.2 indicando un preponderante efecto. Los estudiantes del aula del sol valoraron, en un promedio de 4.7, que el objetivo de ecología humana que más benefició sus relaciones intrapersonales fue el conocimiento de sí mismo y la autoestima. Los objetivos de ecología humana que más beneficiaron sus relaciones interpersonales fueron el cuidado del ambiente, el trabajo en equipo y la empatía (Figura 5).

Figura 5. Medida en que los objetivos de Ecología Humana beneficiaron las relaciones intra e interpersonales

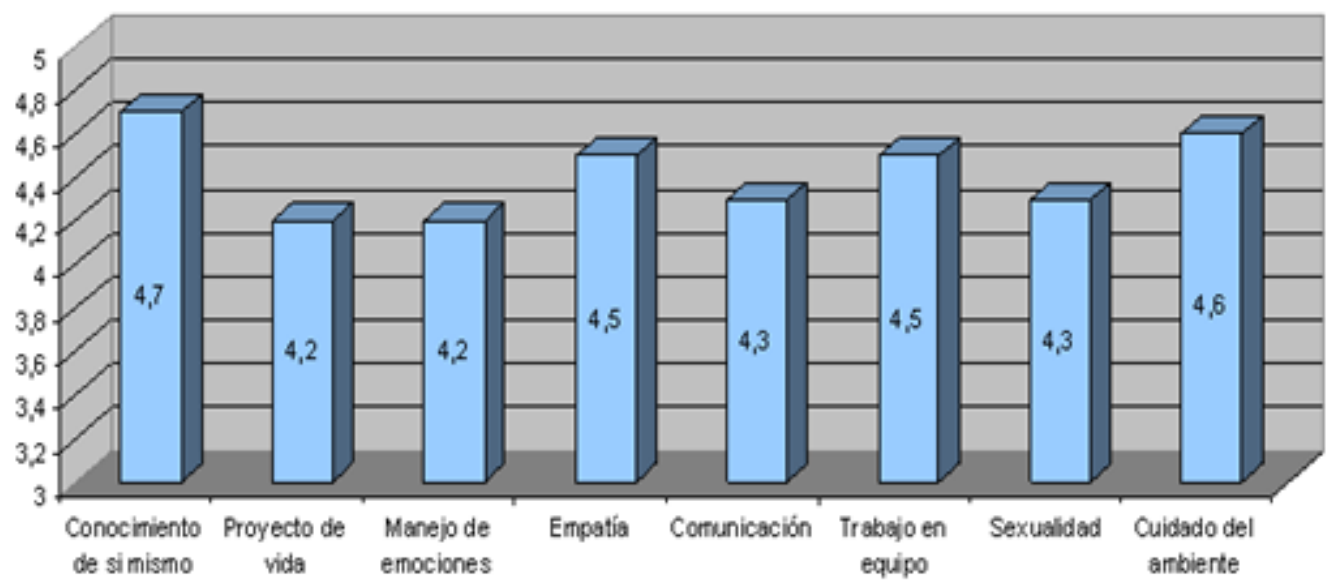

Finalmente es importante mencionar varios aspectos dentro de la interpretación del diario de campo:

La ecología humana respondió a la necesidad de los docentes y coordinadores del aula del sol para vincular los estudiantes a su semana, motivándolos e integrándolos con las actividades académicas.

Se evidenció el cambio de actitud de los jóvenes en el desarrollo de los talleres. La rebeldía y la agresividad disminuyeron en todos los estudiantes que llevaron el proceso. Sus participaciones lo hicieron aún más enriquecedor.

Los jóvenes aprovecharon el espacio para reflexionar, divertirse, opinar, formarse y tener razones para continuar avanzando en su proceso. Se motivaron mutuamente buscando aprender del pasado, aprovechando el presente y sembrando un excelente futuro. Este compartir generó gran confianza entre ellos, expresaron libremente sus pensamientos y sus proyectos. Gracias a esto se pudo partir de la 
realidad para intervenir adecuadamente en su proceso. Se encontraron vías para hallar la verdadera esencia del ser humano y se plantearon otras posibilidades en la vida para lograr la verdadera felicidad.

Hoy los jóvenes continúan conociéndose a sí mismos, encuentran sus debilidades y fortalezas; siguen soñando con una mejor vida, pero lo más importante es que se han enterado de que los sueños se alcanzan cuando todos los días se lucha por lograrlos.

\section{CONCLUSIONES}

Los estudiantes del aula del sol desarrollan y transforman su convivencia humana, las relaciones del $100 \%$ de los estudiantes mejoran con sus compañeros, sus docentes y su entorno.

El trabajo en equipo visualiza el fortalecimiento de las relaciones interpersonales dentro del aula del sol. El $79 \%$ de los estudiantes consideran sus relaciones interpersonales muy buenas, la razón de ser de ello se explica en que trabajan en equipo. El $72 \%$ de los estudiantes encuentran éste como la razón de ser en el mejoramiento de sus relaciones.

Los objetivos de la Ecología Humana influyen en los estudiantes del aula del sol. El conocimiento de sí mismo, la autoestima, el cuidado del ambiente, trabajar en equipo y la empatía son los objetivos más influyentes en sus relaciones.

Se manifiesta el cambio de actitud en todos los jóvenes del aula del sol dentro su proceso formativo. La rebeldía y la agresividad disminuyen en los estudiantes. Se motivan fácilmente y su participación hace enriquecedora la estadía con y entre ellos.

Trabajadoras sociales, psicólogos, abogado y docente coordinan el aula del sol. A su vez, el aula del sol los ha influenciado, les ha permitido comprender diferentes ambientes y procesos humanos dentro de las dimensiones psicosociales, cognitivas, emocionales, familiares, gestar espacios de vida juvenil reconociendo al estudiante como el ser humano que es, entender y valorar el ser humano resaltando sus potencialidades y la fuerza de su transformación.

\section{AGRADECIMIENTOS}

El autor expresa sus agradecimientos a los docentes María Luisa Álvarez Mejía y Jairo Andrés Velásquez Sarria, al aula del sol, a su equipo coordinador y a todas aquellas personas que tuvieron que ver con esta investigación. Gracias por el apoyo y la motivación.

\section{BIBLIOGRAFÍA}

- RESTREPO, Luis Carlos. (1996). Ecología humana: una estrategia de intervención cultural. Santafé de Bogotá: San Pablo.

- SAAVEDRA QUESADA, Juan Antonio. (2004). El corazón tiene razón. Las palmas de Gran Canaria: Ediciones Anaga.

- WILCHES-ChAUX, Gustavo. (1999). De nuestros deberes para con la vida. Popayán: Corporación Autónoma regional del Cauca (CRC).

\section{WEBGRAFÍA:}

- $\quad$ TAPIA FORTUNATO, Juan José. nd/2.009. URL http://www.ecologiadelserhumano.com (visitado 2009/06/03). 
- SAAVEDRA QUESADA, Juan Antonio. nd/2.009. URL http://www.ehcanarias.com (visitado 2009/06/03).

1. Licenciado en biología y química con formación médica. Docente del programa capacitación de educadores en ejercicio del Departamento de Estudios Educativos de la Universidad de Caldas.Email: osanalme@hotmail.com

2. Investigación realizada por Natalia Springer, asesora de la organización internacional de migraciones (O.I.M.).

3. Ministerio de Educación Nacional. Resolución No 2620 del 1 de septiembre de 2004.

4. Se reconoce la importancia de la institución educativa, pero no se menciona su nombre por la seguridad de sus estudiantes. 